\title{
Decreasing the spectral radius of a graph by link removals
}

\author{
Piet Van Mieghem, ${ }^{*}$ Dragan Stevanović, ${ }^{\dagger}$ Fernando Kuipers, Cong Li, Ruud van de Bovenkamp, \\ Daijie Liu, and Huijuan Wang \\ Delft University of Technology, Delft, The Netherlands
}

(Received 15 April 2011; published 6 July 2011)

\begin{abstract}
The decrease of the spectral radius, an important characterizer of network dynamics, by removing links is investigated. The minimization of the spectral radius by removing $m$ links is shown to be an NP-complete problem, which suggests considering heuristic strategies. Several greedy strategies are compared, and several bounds on the decrease of the spectral radius are derived. The strategy that removes that link $l=i \sim j$ with largest product $\left(x_{1}\right)_{i}\left(x_{1}\right)_{j}$ of the components of the eigenvector $x_{1}$ belonging to the largest adjacency eigenvalue is shown to be superior to other strategies in most cases. Furthermore, a scaling law where the decrease in spectral radius is inversely proportional to the number of nodes $N$ in the graph is deduced. Another sublinear scaling law of the decrease in spectral radius versus the number $m$ of removed links is conjectured.
\end{abstract}

DOI: 10.1103/PhysRevE.84.016101

PACS number(s): 89.75.Hc, 89.20.-a

\section{INTRODUCTION}

The largest eigenvalue $\lambda_{1}(A)$ of the adjacency matrix $A$, called the spectral radius of the graph, plays an important role in dynamic processes on graphs, such as, e.g., virus spread [1]. In a susceptible-infectious-susceptible (SIS) type of network infection, the steady-state ${ }^{1}$ infection of the network is determined by a phase transition at the epidemic threshold $\tau_{c}=\frac{1}{\lambda_{1}(A)}$ : When the effective infection rate $\tau>\tau_{c}$, the network is infected, whereas below $\tau_{c}$, the network is virus free. Beside virus spread, the same type of phase-transition threshold [2] in the coupling strength $g_{c} \sim \frac{1}{\lambda_{1}(A)}$ occurs in a network of coupled oscillators.

Motivated by a $\frac{1}{\lambda_{1}(A)}$ threshold separating two different phases of a dynamic process on a network, we want to change the network in order to enlarge the network's epidemic threshold $\tau_{c}$, or, equivalently, to lower $\lambda_{1}(A)$. Removing nodes is often too drastic, ${ }^{2}$ and, therefore, we concentrate here mainly on the problem of removing $m$ links from a graph $G$ with $N$ nodes and $L$ links. We are searching for a strategy so that, after removing $m$ links, $\lambda_{1}$ is minimal. Earlier Restrepo et al. [4] have initiated an instance of this problem: "How does $\lambda_{1}$ decrease when links are removed?" They introduced a new graph metric, called the dynamical importance $I_{x}=\frac{\lambda_{1}(A)-\lambda_{1}(A \backslash\{x\})}{\lambda_{1}(A)}$, where $x$ denotes the removal either of a link $x=l$ or of a node $x=n$. The dynamical

\footnotetext{
*Faculty of Electrical Engineering, Mathematics and Computer Science, P. O. Box 5031, NL-2600 GA Delft, The Netherlands; P.F.A.VanMieghem@tudelft.nl

${ }^{\dagger}$ Faculty of Mathematics, Natural Sciences and Information Technology, University of Primorska, Glagoljaska 8, 6000 Koper, Slovenia, and Faculty of Sciences and Mathematics, University of Nis, Visegradska 33, RS-18000 Nis, Serbia.

${ }^{1}$ In the exact SIS model, the steady state is the healthy state, which is the only absorbing state in the Markov process. However, in networks of realistic size $N$, this steady state is reached only after an unrealistically long time. The steady state in the $N$-intertwined virus spread model refers to the metastable state, which is reached exponentially rapidly and which reflects real epidemics more closely.

${ }^{2}$ The influence of the addition of a node in $G$ to the spectrum of $A$ is discussed in Ref. [3], art. 60].
}

importance was further investigated by Milanese et al. [5]. Both Restrepo et al. and Milanese et al. have approached the problem by using perturbation theory. However, they did not consider optimality of their removal strategy.

In this paper, we complement their study by first showing in Sec. II that the Link Spectral Radius Minimization (LSRM) problem and the Nodal Spectral Radius Minimization (NSRM) problem, defined in Problem 1 and Problem 3, are NP-hard, which means in practice that an optimal solution in a large network cannot be computed and that good approximate algorithms or heuristics need to be devised. The NP-completeness of LSRM and NSRM is demonstrated by reducing the problem to an equivalent problem, namely, finding a Hamiltonian path in a graph that is known to be NP-complete [6]. Since LSRM and NSRM are NP-complete, we cannot hope to find exact analytic formulas for the decrease in the spectral radius. However, in Sec. III we provide a general analytic description, bounds, and several lemmas, and we study the effect of node and link removal on closed walks and the influence of assortativity on the spectral radius. This developed theory direct us to find good heuristics. Section IV proposes eight different strategies (or heuristics) for removing one link in a network, and these strategies are benchmarked with the optimal strategy via extensive simulations. The removal of the link $l$ between nodes $i$ and $j$ with highest product $\left(x_{1}\right)_{i}\left(x_{1}\right)_{j}$ of the eigenvector components belonging to the largest eigenvalue $\lambda_{1}(A)$ of the adjacency $A$ is demonstrated to be the best heuristic. However, it is not always the best heuristic when more than one link is removed, as illustrated in Figs. 1-3. The scaling law (18) for removing one link in Sec. V demonstrates, presumably for all graphs, that a decrease in $\lambda_{1}$ is inversely proportional to the size $N$ of the graph. Hence, small graphs show the effect of link removals on $\lambda_{1}$ more clearly than large graphs. The scaling law (15) is much less accurately known but indicates a sublinear decrease in $\lambda_{1}$ with the number $m$ of removed links. We also claim that the optimal way to remove $m$ links is to make the resulting graph as regular as possible, because a regular graph has the lowest spectral radius among all graphs with $N$ nodes and $L$ links.

Another type of strategy to prevent the outbreak of a virus is to quarantine infected nodes. Omic et al. [7] have studied immunization via modularity partitioning, where 

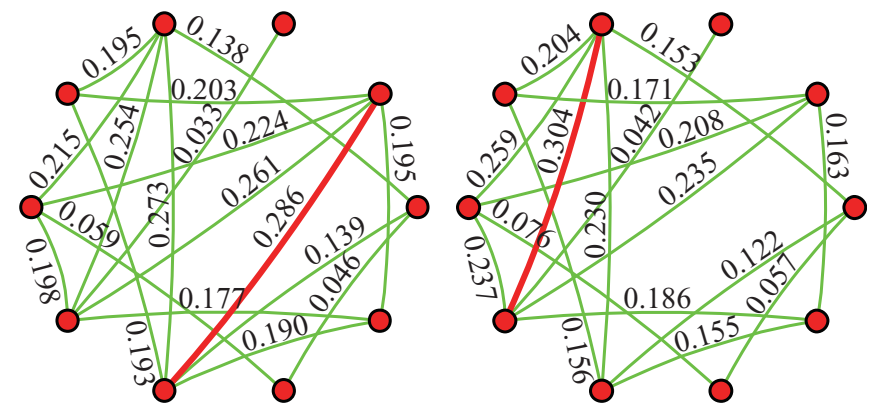

Step 1

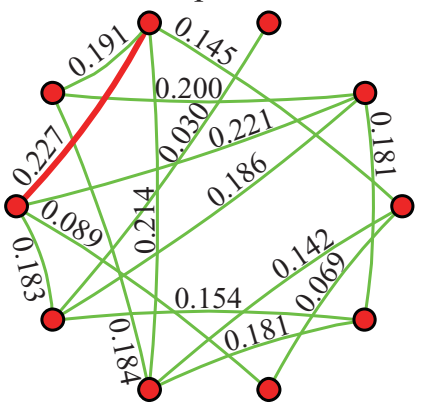

Step 3

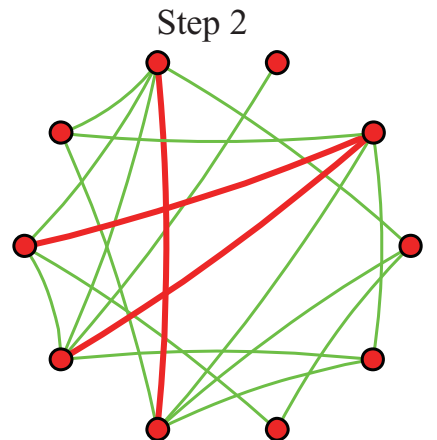

Optimal

FIG. 1. (Color online) An example of a graph with $N=10$ nodes, where none of the links in the greedy approach appears in the optimal set of links. The numbers indicate the change in largest eigenvalue $\lambda_{1}(A)-\lambda_{1}(A \backslash\{l\})$ after removal of link $l$.

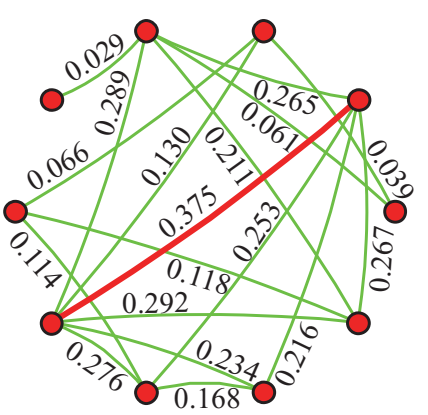

Step 1

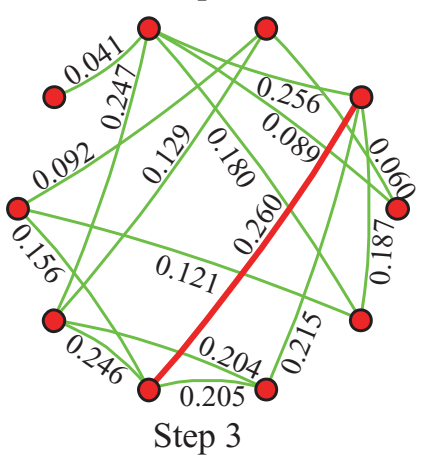

FIG. 2. (Color online) An example of a graph with $N=10$ nodes, where only 1 link in the greedy approach appears in the optimal set of links.
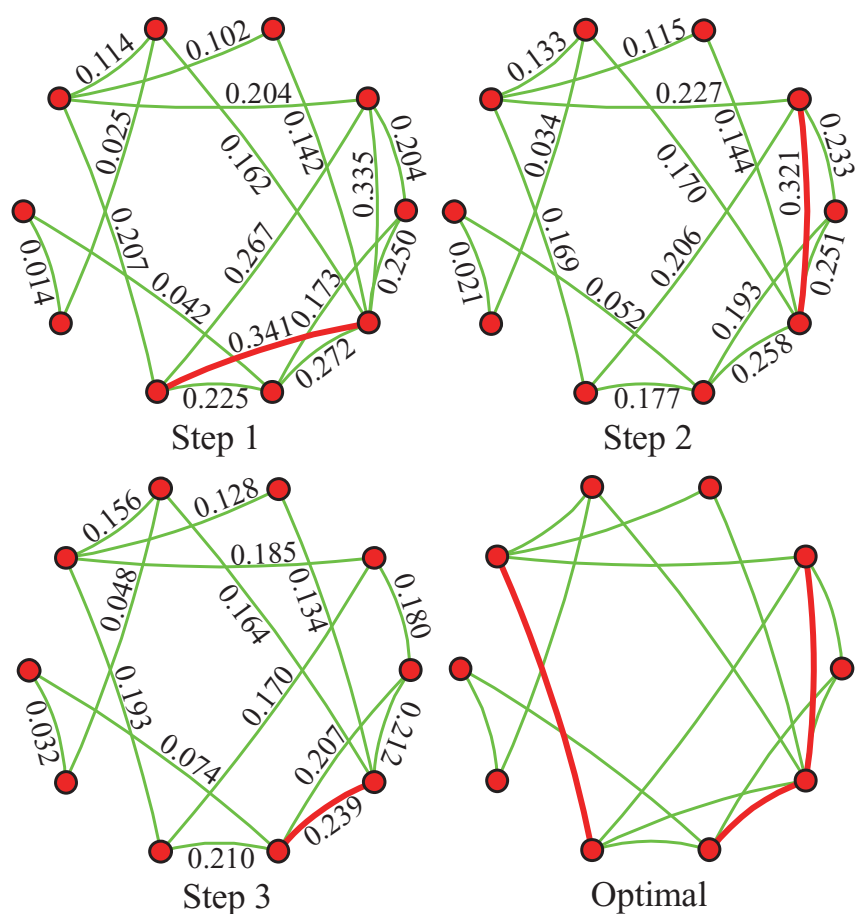

FIG. 3. (Color online) An example of a graph with $N=10$ nodes, wheretwo links in the greedy approach appear in the optimal set of links.

inter-community links are removed such that intracommunity communication is preserved. Taylor and Restrepo [8] investigated the effect of adding a subgraph to a network on its largest adjacency eigenvalue $\lambda_{1}$. Inspired by network synchronization, Watanabe and Masuda [9] have investigated a similar problem as the NSRM but with a different object function: remove nodes in a graph to maximize the second smallest eigenvalue of the Laplacian of the graph, also coined the algebraic connectivity [3]. They have presented several strategies comparable to ours and also found that the eigenvector strategy performed overall the best. Related to Ref. [9], but based on a weighted, asymmetric Laplacian of a graph, Nishikawaa and Mottera [10] point to the nontrivial effect of link removals on network synchronization.

\section{THE SPECTRAL RADIUS MINIMIZATION PROBLEM IS NP-HARD}

In this section we prove that optimally decreasing the largest adjacency eigenvalue (the spectral radius) of a graph by a fixed number of link removals is NP-hard. It is widely believed that NP-hard problems cannot be solved exactly in a time complexity that is upper bounded by a polynomial function of the relevant input parameters $(N$ and $L)$. Let us first formulate the Link Spectral Radius Minimization (LSRM) problem precisely:

Problem 1 (Link Spectral Radius Minimization (LSRM) problem). Given a graph $G(\mathcal{N}, \mathcal{L})$ with $N$ nodes and $L$ links, spectral radius $\lambda_{1}(G)$, and an integer number $m<L$, which $m$ links from the graph $G$ need to be removed, such that the spectral radius of the reduced graph $G_{m}$ of $L-m$ links has 
the smallest spectral radius out of all possible graphs that can be obtained from $G$ by removing $m$ links?

Theorem 1. The LSRM problem is NP-hard.

To prove this theorem, we rely on the following lemmas, but first we need the definition of a path $P_{h}$ with $h$ hops or links. A path $P_{h}$ with $h$ hops starting from a node $n_{0}$ and ending at node $n_{h}$ is defined as $P_{h}=n_{0} \sim n_{1} \sim n_{2} \sim \ldots \sim n_{h-1} \sim n_{h}$, where each link $n_{i} \sim n_{j}$ between nodes $n_{i}$ and $n_{j}$ as well as each node $n_{i}$ occurs once in the sequence defining the path $P_{h}$, in contrast to a walk $W_{h}=n_{0} \sim n_{1} \sim n_{2} \sim \ldots \sim$ $n_{h-1} \sim n_{h}$ with $h$ hops, where a node $n_{i}$ can appear more than once.

Lemma 1. The path $P_{N-1}$ visiting $N$ nodes has a strictly smaller spectral radius than all other connected graphs with $N$ nodes. Furthermore, $\lambda_{1}\left(P_{N-1}\right)=2 \cos \left(\frac{\pi}{N+1}\right)$.

Proof: Ref. [11], p. 21], Ref. [3], p. 125]

Lemma 2. The eigenvalues of a disconnected graph are composed of the eigenvalues (including multiplicities) of its connected components.

Proof: Ref. [3], art. 80, pp 73-74]

Lemma 3. Among all possible graphs of $N$ nodes and $N-1$ links, the path $P_{N-1}$ visiting $N$ nodes has the smallest spectral radius.

Proof: A connected graph of $N$ nodes and $N-1$ links is a tree, of which the path is a special case. According to Lemma 1 the path has a spectral radius strictly smaller than 2 , which is the smallest spectral radius possible in connected graphs. Hence, we need to demonstrate that the lemma also holds for disconnected graphs. For ease of presentation, we assume that the disconnected graph consists of two connected components $A_{1}$ and $A_{2}: A_{1}$ of $x$ nodes and $A_{2}$ of $N-x$ nodes. Our arguments also apply to multiple connected components. Now, $A_{1}$ contains at least $x-1$ links, otherwise it is not a connected component, and $A_{2}$ contains at least $N-x-1$ links. Since the sum of these links equals $N-2$, either $A_{1}$ or $A_{2}$ must contain one extra link, thereby creating a cycle in that component. A graph that contains a cycle (i.e., which is not a tree) has a spectral radius larger than or equal to two. This component will, according to Lemma 2, contribute to an overall spectral radius that is larger than that of a path, which is smaller than 2 .

To prove Theorem 1, we will use the NP-complete Hamiltonian path problem [6].

Problem 2 (Hamiltonian path problem). Given a graph $G(\mathcal{N}, \mathcal{L})$ with $N$ nodes and $L$ links, a Hamiltonian path is a path that visits every node exactly once. The Hamiltonian path problem is to determine if $G$ contains a Hamiltonian path.

We are now ready to prove Theorem 1:

Proof: In our proof we will demonstrate that if we could solve the LSRM problem in polynomial time, then we would also be able to settle the NP-complete Hamiltonian path problem. Assume we have a graph $G$ of $L=N-1+m$ links. Removing $m$ links will result in a graph $G_{m}$ of $N-1$ links. According to Lemma 3, a path is the only graph structure of $N-1$ links that has the smallest largest adjacency eigenvalue and that eigenvalue equals $\lambda_{1}=2 \cos \left(\frac{\pi}{N+1}\right)$. Moreover, a path of $N-1$ links in a graph of $N$ nodes, is a Hamiltonian path. If, after solving the LSRM problem, we obtain $\lambda_{1}=2 \cos \left(\frac{\pi}{N+1}\right)$ (smaller is not possible), then we have found a Hamiltonian path $\left(G_{m}\right)$. If $\lambda_{1}>2 \cos \left(\frac{\pi}{N+1}\right)$, then the original graph $G$ does not contain a Hamiltonian path. The LSRM problem is therefore at least as hard as the Hamiltonian path problem.

We have to interpret Theorem 1 with care. Computing the largest eigenvalue can be done in polynomial time. Consequently the number of possible combinations ${ }_{m}^{L}$ of $m$ links that we could check (by computing in polynomial time the largest eigenvalue of the graph $G_{m}$ resulting after the removal of that specific set of links) is bounded by $O\left(L^{m}\right)$, which is a polynomial function in $L$. For instance, if $m=1$, by checking the spectral radius reduction induced by the removal of each of the $L$ links, we can obtain a solution with a complexity of $L$ times the complexity of computing the largest eigenvalue. However, in that case $m$ is fixed and not part of the input $N, L, m$ as defined in problem 1 . In other words, $m$ should have been replaced with a fixed integer number in the problem definition to make it clear that $m$ is not part of the input and that its fixed value holds for all problem instances. In problem $1, m$ is part of the input and, as in our proof, may, for instance, depend on the number of nodes and links (it makes sense to remove more links in larger networks). The previous argument therefore does not apply to problem 1, which is NP-hard as proved in Theorem 1. In fact, in our proof $m=L-N+1$ so that the worst-case complexity of checking all possibilities is $O\left(L^{L-N+1}\right)$, which is now clearly nonpolynomial in the input $N, L, m$. Similar NP-complete problems, in which the input does not only rely on $N$ and $L$, but also on another metric $k$, are the Independent Set problem (defined in problem 4 below) and the Disjoint Connecting Paths problem [6], where $k$ mutually node-disjoint paths need to be found between $k$ corresponding source-destination pairs. This problem also can be solved in polynomial time if $k$ is fixed and thus not part of the input [12], while it is NP-complete if $k$ is part of the input. In general, NP-complete problems that can be solved by algorithms, which are exponential only in the size of a fixed parameter while polynomial in the size of the (remaining) input, are called fixed-parameter tractable, because those problems can be solved efficiently for small values of the fixed parameter.

As an example to illustrate the NP-completeness of the LSRM problem, Figs. 1-3 show, in a topology of $N=10$ nodes and $m=3$ link removals, that the "best single step strategy" is not always optimal in the end. The "best single step strategy" consists of removing the link that lowers $\lambda_{1}(A)-$ $\lambda_{1}\left(A_{1}\right)=y_{1}$ most in the first step. Next, in the second step, the link that lowers $\lambda_{1}\left(A_{1}\right)-\lambda_{1}\left(A_{2}\right)=y_{2}$ most is removed, and finally, in the third step, the link that lowers $\lambda_{1}\left(A_{2}\right)-\lambda_{1}\left(A_{3}\right)=$ $y_{3}$ most is removed. The optimal situation depicts the removal of $m=3$ for which $\lambda_{1}(A)-\lambda_{1}\left(A_{3}\right)=y^{*}$ is maximal. Hence, $y_{1}+y_{2}+y_{3} \leqslant y^{*}$.

In addition, $10^{6}$ instances of Erdős-Rényi (ER) random graphs with $N=10$ and link density $p=\frac{2 \ln N}{N}$ have been generated. In each instance, the "best single step strategy" and the global optimum have been computed. In 63185 $(6.3 \%)$ instances, there was no overlap in links, in 332262 (33.2\%) ER graphs, there was one link in common, in 97944 (9.8\%) ER graphs, we found two links in common, and in the remaining 506609 (50.7\%) ER graphs, all three links in the "best single step strategy" were the same as in the global optimum. Moreover, Fig. 4 illustrates that the global optimum is not always unique. The global optimum may not be unique, 

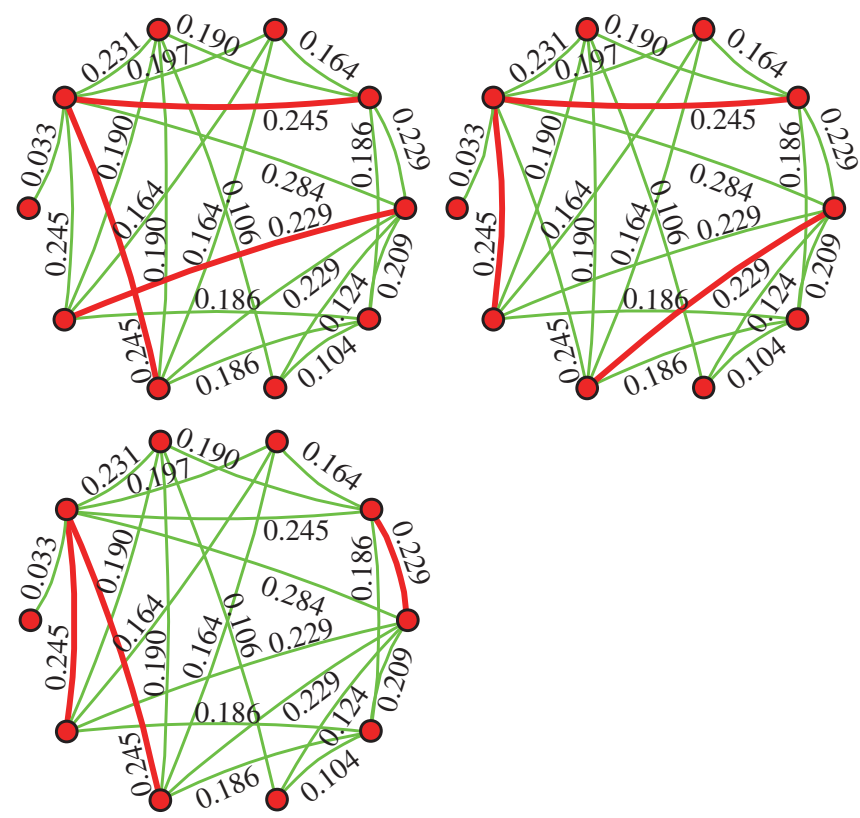

FIG. 4. (Color online) An example where the global optimum is not unique. The original $\lambda_{1}(A)=5.065310$, and, after removal of three links, the smallest largest eigenvalue is $\lambda_{1}\left(A_{3}\right)=4.312414$. Consequently, the largest $\lambda_{1}(A)-\lambda_{1}\left(A_{3}\right)=0.752896$ is obtained after removal of the three red links.

as it is possible that the removals of different sets of $m$ links will lead to cospectral or even isomorphic smaller graphs, as indicated in Fig. 4.

The minimum number $m$ of links that need to be removed from $G$ to ensure that $\lambda_{1}$ in $G_{m}$ is lowered below some given value $\xi$ is

$$
m \leqslant L-\frac{\xi^{2}+N-1}{2},
$$

which is derived from the bound [3], (3.48) on p. 54], due to Yuan Hong [13],

$$
\lambda_{1} \leqslant \sqrt{2 L-N+1}
$$

for connected graphs; else $\lambda_{1} \leqslant \sqrt{2 L\left(1-\frac{1}{N}\right)}$.

\section{A. Link versus node removal}

Removing nodes to maximally lower the largest eigenvalue may seem an easier problem than removing links. For, when we remove the highest degree node, $\lambda_{1}(A)$ is likely reduced most (because $L$ is reduced most). This suggestion follows from bounds in Ref. [3], p. 48] and the bounds

$$
\frac{2 L}{N} \sqrt{1+\frac{\operatorname{Var}[D]}{(E[D])^{2}}} \leqslant \lambda_{1} \leqslant \min \left\{\sqrt{\frac{2 L(N-1)}{N}}, d_{\max }\right\},
$$

where $D$ is the degree of an arbitrary node in $G$. Unfortunately, this intuition is wrong. The eigenvalues of the adjacency matrix $A_{l(G)}$ of the line graph $l(G)$ of $G$ and $A$ are related [3], (2.9) on p. 20]. Since links in $G$ are nodes in $l(G)$, and since there is a one-to-one relation between $l(G)$ and $G$, removing nodes in $l(G)$ according to a certain strategy results in a corresponding strategy for removing links in $G$. Since the link spectral radius minimization (LSRM) problem is NP-hard (Theorem 1), the problem of removing $m$ nodes from a graph $G$ is NP-hard as well. We will provide a proof for general graphs and subsequently demonstrate that it is also NP-complete in the subclass of line graphs. Let us first formally define the problem:

Problem 3 (Nodal Spectral Radius Minimization (NSRM) problem). Given a graph $G(\mathcal{N}, \mathcal{L})$ with $N$ nodes and $L$ links, spectral radius $\lambda_{1}(G)$, and an integer number $m<N$, which $m$ nodes from the graph $G$ need to be removed, such that the spectral radius of the reduced graph $G_{m}$ of $N-m$ nodes has the smallest spectral radius out of all possible graphs that can be obtained from $G$ by removing $m$ nodes?

Theorem 2. The NSRM problem is NP-hard.

We provide a proof by reducing the NP-complete independent set problem [6] to NSRM.

Problem 4 (Independent set problem) Given a graph $G(\mathcal{N}, \mathcal{L})$ with $N$ nodes and $L$ links and a positive integer $k \leqslant N$, is there a subset $\mathcal{N}^{\prime} \subseteq \mathcal{N}$, such that $\left|\mathcal{N}^{\prime}\right| \geqslant k$ and such that no two nodes in $\mathcal{N}^{\prime}$ are joined by a link in $\mathcal{L}$ ?

Proof of Theorem 2: The lowest spectral radius of a graph equals $\lambda_{1}(G)=0$, which is obtained for a graph without any links. Removing nodes that are not part of an independent set will result in an independent set of nodes that are not linked to each other. Hence, to solve the independent set problem it suffices to remove $m=N-k$ nodes from the graph $G$, such that the spectral radius of the reduced graph $G_{m}$ is smallest possible. If we get $\lambda_{1}\left(G_{m}\right)=0$, then $G_{m}$ constitutes an independent set of $k$ nodes. If $\lambda_{1}\left(G_{m}\right)>0$, then no independent set with at least $k$ nodes exists.

Line graphs are a specific class of graphs, and not all problems that are NP-complete for general graphs are also NP-complete for line graphs (e.g., according to Roussopoulos [14], the clique problem is not hard in line graphs, while it is an NP-complete problem in general). Hence, we proceed to demonstrate that the NSRM problem remains NP-hard in line graphs. We use similar arguments as for the proof of Theorem 1. A Hamiltonian path in the graph $G$ corresponds to a path of $N-1$ nodes in the line graph $l(G)$ of $G$. A line graph $l(G)$ contains $L$ nodes and can be generated in polynomial time from $G$. According to Lemma 1, the graph structure of $N-1$ nodes that has the smallest largest eigenvalue is the path. Hence, removing $L-N+1$ nodes from the line graph $l(G)$ such that the spectral radius is reduced most should correspond to a path of $N-1$ nodes (if it exists), which corresponds to a Hamiltonian path in $G$. Solving the NSRM problem in a line graphs $l(G)$ is therefore as hard as finding Hamiltonian paths in the corresponding graph $G$.

Finally, let $l$ be the removed link that maximizes $\lambda_{1}(G)-$ $\lambda_{1}(G \backslash\{l\})$. Let the node $n$ be the transform of link $l$ in the line graph $l(G)$. Then $\lambda_{1}(l(G))-\lambda_{1}(l(G) \backslash\{n\})$ is not always the maximum. Simulations on 100 Erdős-Rényi random graphs show the "success rate,"the percentage of graphs in which the best link $l$ in $G$ corresponds to the best node $n$ in $l(G)$ in Table I.

\section{SPECTRAL GRAPH THEORY}

We derive a theoretical underpinning to deduce the best heuristic for the LSRM problem. We first introduce the notation. Let $x_{1}$ be the eigenvector of $A$ belonging to $\lambda_{1}(A)$ in 
TABLE I. Success rate in line graphs.

\begin{tabular}{llcc}
\hline \hline & \multicolumn{3}{c}{$p$} \\
\cline { 2 - 4 }$N$ & 0.1 & 0.2 & 0.3 \\
\hline 10 & $67 \%$ & $80 \%$ & $83 \%$ \\
20 & $65 \%$ & $76 \%$ & $81 \%$ \\
30 & $59 \%$ & $76 \%$ & $81 \%$ \\
40 & $70 \%$ & $79 \%$ & $81 \%$ \\
50 & $63 \%$ & $75 \%$ & $82 \%$ \\
60 & $67 \%$ & $82 \%$ & $86 \%$ \\
\hline \hline
\end{tabular}

the original graph $G$ and normalized such that $x_{1}^{T} x_{1}=1$. The graph $G$ contains $N$ nodes and $L$ links. Let $\mathcal{M}_{m}$ denote the set of the $m$ links that are removed from $G$ and $G_{m}=G \backslash \mathcal{M}_{m}$ is the resulting graph after the removal of $m$ links from $G$. We denote the adjacency matrix of $G_{m}$ by $A_{m}$, which is still a symmetric matrix. Similarly, let $w_{1}$ be the normalized eigenvector of $A_{m}$ corresponding to $\lambda_{1}\left(A_{m}\right)$ in the graph $G_{m}$. By the Perron-Frobenius theorem [3], all components of $x_{1}$ and $w_{1}$ are nonnegative (positive if the corresponding graph is connected).

Let $e_{j}$ be a base vector in the $N$-dimensional space, where the $i$ th component equals $\left(e_{j}\right)_{i}=\delta_{i j}$ and $\delta_{i j}$ is the Kronecker delta, i.e., $\delta_{i j}=1$ if $i=j$, and otherwise, $\delta_{i j}=0$. Then the adjacency matrix that represents the single link between nodes $i$ and $j$ equals

$$
\hat{A}_{i j}=e_{i} e_{j}^{T}+e_{j} e_{i}^{T} .
$$

Thus, $\hat{A}_{i j}$ equals the zero matrix, except that $\left(\hat{A}_{i j}\right)_{i j}=$ $\left(\hat{A}_{i j}\right)_{j i}=1$. Clearly, $\operatorname{det}\left(\hat{A}_{i j}-\lambda I\right)=(-1)^{N} \lambda^{N-2}\left(\lambda^{2}-1\right)$, such that the largest eigenvalue of $\hat{A}_{i j}$ is 1 . Also, for any vector $z$,

$$
\begin{aligned}
z^{T} \hat{A}_{i j} z & =z^{T}\left(e_{i} e_{j}^{T}+e_{j} e_{i}^{T}\right) z \\
& =z^{T} e_{i} e_{j}^{T} z+z^{T} e_{j} e_{i}^{T} z=2 z_{i} z_{j}
\end{aligned}
$$

By invoking $0 \leqslant\left(z_{i}-z_{j}\right)^{2}$, we observe that $2 z_{i} z_{j} \leqslant z_{i}^{2}+$ $z_{j}^{2} \leqslant \sum_{i=1}^{N} z_{i}^{2}=z^{T} z$. Hence, when considering normalized vectors such that $z^{T} z=\|z\|_{2}^{2}=1$, we obtain the upper bound

$$
2 z_{i} z_{j} \leqslant 1 \text {. }
$$

After these preliminaries, we now embark on the problem.

\section{A. The difference $\lambda_{1}(A)-\lambda_{1}\left(A_{m}\right)$}

With the normalization $x_{1}^{T} x_{1}=1$ and $w_{1}^{T} w_{1}=1$, the Rayleigh relations [3] become

$$
\begin{gathered}
\lambda_{1}(A)=x_{1}^{T} A x_{1}, \\
\lambda_{1}\left(A_{m}\right)=w_{1}^{T} A_{m} w_{1} .
\end{gathered}
$$

Writing out the quadratic form

$$
\begin{aligned}
\lambda_{1}(A) & =x_{1}^{T} A x_{1}=\sum_{i=1}^{N} \sum_{j=1}^{N} a_{i j}\left(x_{1}\right)_{i}\left(x_{1}\right)_{j} \\
& =2 \sum_{i=1}^{N} \sum_{j=i+1}^{N} a_{i j}\left(x_{1}\right)_{i}\left(x_{1}\right)_{j}
\end{aligned}
$$

$$
=2 \sum_{l=(i \sim j) \in \mathcal{L}}\left(x_{1}\right)_{i}\left(x_{1}\right)_{j}=2 \sum_{l=1}^{L}\left(x_{1}\right)_{l^{+}}\left(x_{1}\right)_{l^{-}},
$$

where a link $l$ joins the nodes $l^{+}$and $l^{-}$and shows that $\lambda_{1}(A)$ can be written as a sum of positive products over all links in the graph $G$.

We now provide a general bound on the difference between the largest eigenvalues in $G$ and $G_{m}=G \backslash \mathcal{M}_{m}$, where $m$ links are removed.

Lemma 4. For any graph $G$ and $G_{m}=G \backslash \mathcal{M}_{m}$, it holds that

$$
\begin{aligned}
2 \sum_{l \in \mathcal{M}_{m}}\left(w_{1}\right)_{l^{+}}\left(w_{1}\right)_{l^{-}} & \leqslant \lambda_{1}(A)-\lambda_{1}\left(A_{m}\right) \\
& \leqslant 2 \sum_{l \in \mathcal{M}_{m}}\left(x_{1}\right)_{l^{+}}\left(x_{1}\right)_{l^{-}},
\end{aligned}
$$

where $x_{1}$ and $w_{1}$ are the eigenvectors of $A$ and $A_{m}$ corresponding to the largest eigenvalues $\lambda_{1}(A)$ and $\lambda_{1}\left(A_{m}\right)$, respectively, and where a link $l$ joins the nodes $l^{+}$and $l^{-}$.

Proof: Since $A_{m}=A-\sum_{l \in \mathcal{M}_{m}} \hat{A}_{l^{+} l^{-}}$where the left-hand side (or start) of the link $l$ is the node $l^{+}$and the right-hand side (or end) of the link $l$ is the node $l^{-}$and with the normalization $x_{1}^{T} x_{1}=1$, the Rayleigh relations [3] yield

$$
\begin{aligned}
\lambda_{1}(A) & =x_{1}^{T} A x_{1}=x_{1}^{T}\left(A_{m}+\sum_{l \in \mathcal{M}_{m}} \hat{A}_{l^{+} l^{-}}\right) x_{1} \\
& =x_{1}^{T} A_{m} x_{1}+\sum_{l \in \mathcal{M}_{m}} x_{1}^{T} \hat{A}_{l^{+} l^{-}} x_{1} .
\end{aligned}
$$

Using (4) yields $x_{1}^{T} \hat{A}_{l^{+} l^{-}} x_{1}=2\left(x_{1}\right)_{l^{+}}\left(x_{1}\right)_{l^{-}}$, and we arrive at

$$
\lambda_{1}(A)=x_{1}^{T} A_{m} x_{1}+2 \sum_{l \in \mathcal{M}_{m}}\left(x_{1}\right)_{l^{+}}\left(x_{1}\right)_{l^{-}} .
$$

The Rayleigh principle states that, for any normalized vector $w$ with $w^{T} w=1$, it holds that $w^{T} A w \leqslant \lambda_{1}(A)$ and equality is attained only if $w$ equals the eigenvector of $A$ belonging to $\lambda_{1}(A)$. Since $x_{1}$ is not the eigenvector of $A_{m}$ belonging to $\lambda_{1}\left(A_{m}\right)$, we have that $x_{1}^{T} A_{m} x_{1} \leqslant \lambda_{1}\left(A_{m}\right)$ and

$$
\begin{aligned}
\lambda_{1}(A) & =x_{1}^{T} A_{m} x_{1}+2 \sum_{l \in \mathcal{M}_{m}}\left(x_{1}\right)_{l^{+}}\left(x_{1}\right)_{l^{-}} \\
& \leqslant \lambda_{1}\left(A_{m}\right)+2 \sum_{l \in \mathcal{M}_{m}}\left(x_{1}\right)_{l^{+}}\left(x_{1}\right)_{l^{-}}
\end{aligned}
$$

from which the upper bound in (6) is immediate. When repeating the analysis from the point of view of $A_{m}$ rather than from $A$, then

$$
\begin{aligned}
\lambda_{1}\left(A_{m}\right) & =w_{1}^{T} A_{m} w_{1}=w_{1}^{T}\left(A-\sum_{l \in \mathcal{M}_{m}} \hat{A}_{l^{+} l^{-}}\right) w_{1} \\
& =w_{1}^{T} A w_{1}-2 \sum_{l \in \mathcal{M}_{m}}\left(w_{1}\right)_{l^{+}}\left(w_{1}\right)_{l^{-}}
\end{aligned}
$$

By invoking the Rayleigh principle again, we arrive at the lower bound.

For connected graphs $G$ and $G_{m}$, it is known that $\lambda_{1}(A)-\lambda_{1}\left(A_{m}\right)>0$ (see Lemma 7 in Ref. [3]). The same 
conclusion also follows from Lemma 4 because the PerronFrobenius theorem [3] states that all vector components of $w_{1}$ (and $x_{1}$ ) are positive in a connected graph $G_{m}$. Lemma 4 indicates that, when those $m$ links are removed that maximize $2 \sum_{l \in \mathcal{M}_{m}}\left(x_{1}\right)_{l^{+}}\left(x_{1}\right)_{l^{-}}$, then the upper bound in (6) is maximal, which may lead to the largest possible difference $\lambda_{1}(A)-$ $\lambda_{1}\left(A_{m}\right)$. However, those removed links do not necessarily also maximize the lower bound $2 \sum_{l \in \mathcal{M}_{m}}\left(w_{1}\right)_{l^{+}}\left(w_{1}\right)_{l^{-}}$. Hence, the greedy strategy of removing consecutively the link $l$ with the highest product $\left(x_{1}\right)_{l^{+}}\left(x_{1}\right)_{l^{-}}$is not necessarily guaranteed to lead to the overall optimum. The fact that the SRM problem is NP-hard, as proved in Sec. II, underlines this remark.

Lemma 8 in Ref. [3] states that

$$
\lambda_{1}(A)-\lambda_{1}\left(A_{m}\right) \leqslant \lambda_{1}\left(A-A_{m}\right)
$$

Since $A-A_{m}=\sum_{l \in \mathcal{M}_{m}} \hat{A}_{l^{+} l^{-}}$, it remains to find a close upper bound for $\lambda_{1}\left(\sum_{l \in \mathcal{M}_{m}} \hat{A}_{l^{+} l^{-}}\right)$. Using the bounds [3], (3.48) on p. 54] gives

$$
\begin{aligned}
\lambda_{1}\left(\sum_{l \in \mathcal{M}_{m}} \hat{A}_{l^{+} l^{-}}\right) \leqslant & \min \left(\sqrt{2 m-N+1}, d_{\max }\left(A-A_{m}\right)\right) 1_{\left\{A-A_{m} \text { is a connected graph }\right\}} \\
& +\min \left(\sqrt{2 m-\frac{2 m}{N}}, d_{\max }\left(A-A_{m}\right)\right) 1_{\left\{A-A_{m} \text { is not a connected graph }\right\} .}
\end{aligned}
$$

In general, it is difficult to find sharper bounds (see, e.g., Refs. [15,16]). If $m=2$, then $\lambda_{1}\left(\sum_{l \in \mathcal{M}_{2}} \hat{A}_{l^{+} l^{-}}\right)=\sqrt{2}$ when the two links are connected, and $\lambda_{1}\left(\sum_{l \in \mathcal{M}_{2}} \hat{A}_{l^{+} l^{-}}\right)=1$ when the two links are disconnected. If $m=1$, then $\lambda_{1}\left(\hat{A}_{l^{+} l^{-}}\right)=1$, and we obtain

$$
\lambda_{1}(A)-\lambda_{1}\left(A_{1}\right) \leqslant 1 .
$$

Lemma 5. For $m=1$ link removed from $G$, equality in (7) is attained only for the graph consisting of the complete graph $K_{N}$ with $N=2$ nodes and a set of disjoint nodes.

Proof: Equality in (7) combined with (6) in Lemma 4 implies that

$$
1=\lambda_{1}(A)-\lambda_{1}\left(A_{1}\right) \leqslant 2\left(x_{1}\right)_{l^{+}}\left(x_{1}\right)_{l^{-}} .
$$

Next, from $2\left(x_{1}\right)_{l^{+}}\left(x_{1}\right)_{l^{-}} \leqslant\left(x_{1}\right)_{l^{+}}^{2}+\left(x_{1}\right)_{l^{-}}^{2} \leqslant x_{1}^{T} x_{1}=1$, we conclude that the equality in (7) holds if and only if $\left(x_{1}\right)_{l^{+}}=$ $\left(x_{1}\right)_{l^{-}}=1 / \sqrt{2}$. Since in such a case $\left(x_{1}\right)_{l^{+}}^{2}+\left(x_{1}\right)_{l^{-}}^{2}=1$, we conclude that all other components of the eigenvector $x_{1}$ are equal to zero. Recall that $x_{1}$ is the principle eigenvector, which, according to the Perron-Frobenius Theory, is positive if $G$ is a connected graph. If $G$ has more than two nodes $(N>2)$, the above argument shows that $G$ must be disconnected, with $K_{2}$ being the unique component with the largest spectral radius. Therefore, the remaining components must be isolated nodes.

\section{Application of perturbation theory to $m$ link removals}

Let $\lambda_{1}>\lambda_{2} \geqslant \cdots \geqslant \lambda_{n}$ be the eigenvalues of $A$, with $x_{1}, x_{2}, \ldots, x_{n}$ the corresponding eigenvectors, which form an orthonormal basis. We apply the general perturbation formulas [17]

$$
\begin{aligned}
x(\zeta)= & x_{1}+\zeta \sum_{k=2}^{N} \frac{x_{k}^{T} B x_{1}}{\lambda_{1}-\lambda_{k}} x_{k}+\zeta^{2} \sum_{m=2}^{N}\left[\frac{\left(x_{1}^{T} B x_{1}\right)\left(x_{m}^{T} B x_{1}\right)}{\lambda_{1}-\lambda_{m}}\right. \\
& \left.-\sum_{k=2}^{N} \frac{\left(x_{k}^{T} B x_{1}\right)\left(x_{m}^{T} B x_{k}\right)}{\lambda_{1}-\lambda_{k}}\right] \frac{x_{m}}{\lambda_{m}-\lambda_{1}}+O\left(\zeta^{3}\right)
\end{aligned}
$$

$$
\begin{aligned}
\lambda(\zeta)= & \lambda_{1}+\zeta x_{1}^{T} B x_{1}+\zeta^{2} \sum_{k=2}^{N} \frac{\left(x_{k}^{T} B x_{1}\right)^{2}}{\lambda_{1}-\lambda_{k}} \\
& +\zeta^{3} \sum_{m=2}^{N}\left[\left(x_{m}^{T} B x_{m}\right)-\left(x_{1}^{T} B x_{1}\right)\right]\left(\frac{x_{1}^{T} B x_{m}}{\lambda_{1}-\lambda_{m}}\right)^{2} \\
& +2 \zeta^{3} \sum_{m=2}^{N} \sum_{k=2}^{m-1} \frac{\left(x_{1}^{T} B x_{m}\right)\left(x_{m}^{T} B x_{k}\right)\left(x_{k}^{T} B x_{1}\right)}{\left(\lambda_{1}-\lambda_{m}\right)\left(\lambda_{1}-\lambda_{k}\right)}+O\left(\zeta^{4}\right)
\end{aligned}
$$

for the matrix $A(\zeta)=A+\zeta B$ by using $B=\sum_{l=1}^{m} \hat{A}_{l^{+} l^{-}}$and $\zeta=-1$. We remark that $|\zeta|=1$ is large for a perturbation to be effective in general.

Using the definition (3) of the matrix $\hat{A}_{l^{+} l^{-}}$, we obtain

$$
\begin{aligned}
x_{k}^{T} \hat{A}_{l^{+} l^{-}} x_{q} & =x_{k}^{T} e_{l^{+}} e_{l^{-}}^{T} x_{q}+x_{k}^{T} e_{l^{-}} e_{l^{+}}^{T} x_{q} \\
& =\left(x_{k}\right)_{l^{+}}\left(x_{q}\right)_{l^{-}}+\left(x_{k}\right)_{l^{-}}\left(x_{q}\right)_{l^{+}}
\end{aligned}
$$

and

$x_{k}^{T} B x_{q}=\sum_{l=1}^{m} x_{k}^{T} \hat{A}_{l^{+} l^{-}} x_{q}=\sum_{l=1}^{m}\left[\left(x_{k}\right)_{l^{+}}\left(x_{q}\right)_{l^{-}}+\left(x_{k}\right)_{l^{-}}\left(x_{q}\right)_{l^{+}}\right]$,

where $x_{k}$ denotes the eigenvector of $A$ belonging to eigenvalue $\lambda_{k}$. From (8), the first-order perturbation for the eigenvector of $A_{m}$ is

$$
x_{1}(\zeta) \simeq x_{1}-\sum_{k=2}^{N} \sum_{l=1}^{m} \frac{\left(x_{k}\right)_{l^{+}}\left(x_{q}\right)_{l^{-}}+\left(x_{k}\right)_{l^{-}}\left(x_{q}\right)_{l^{+}}}{\lambda_{1}-\lambda_{k}} x_{k},
$$

and from (9) the corresponding eigenvalue perturbation, up to second order, is

$$
\begin{aligned}
\lambda_{1}(\zeta)= & \lambda_{1}(A)-2 \sum_{l=1}^{m}\left(x_{1}\right)_{l^{+}}\left(x_{1}\right)_{l^{-}} \\
& +\sum_{k=2}^{N} \frac{\left\{\sum_{l=1}^{m}\left[\left(x_{k}\right)_{l^{+}}\left(x_{1}\right)_{l^{-}}+\left(x_{k}\right)_{l^{-}}\left(x_{1}\right)_{l^{+}}\right]\right\}^{2}}{\lambda_{1}-\lambda_{k}} .
\end{aligned}
$$


Since $\lambda_{1}(\zeta)=\lambda_{1}\left(A_{m}\right)$, the difference in largest eigenvalues equals approximately

$$
\begin{aligned}
\lambda_{1}(A)-\lambda_{1}\left(A_{m}\right) \simeq & 2 \sum_{l=1}^{m}\left(x_{1}\right)_{l^{+}}\left(x_{1}\right)_{l^{-}}-\sum_{k=2}^{N} \\
& \times \frac{\left\{\sum_{l=1}^{m}\left[\left(x_{k}\right)_{l^{+}}\left(x_{1}\right)_{l^{-}}+\left(x_{k}\right)_{l^{-}}\left(x_{1}\right)_{l^{+}}\right]\right\}^{2}}{\lambda_{1}(A)-\lambda_{k}(A)} .
\end{aligned}
$$

Of course, we can also apply the perturbation formula to $A_{m}$ and add $m$ links so that $\zeta=1$. The difference in largest eigenvalues equals approximately

$$
\begin{aligned}
\lambda_{1}(A)-\lambda_{1}\left(A_{m}\right) \simeq & 2 \sum_{l=1}^{m}\left(w_{1}\right)_{l^{+}}\left(w_{1}\right)_{l^{-}}+\sum_{k=2}^{N} \\
& \times \frac{\left\{\sum_{l=1}^{m}\left[\left(w_{k}\right)_{l^{+}}\left(w_{1}\right)_{l^{-}}+\left(w_{k}\right)_{l^{-}}\left(w_{1}\right)_{l^{+}}\right]\right\}^{2}}{\lambda_{1}\left(A_{m}\right)-\lambda_{k}\left(A_{m}\right)} .
\end{aligned}
$$

The proof of Lemma 4 indicates that the difference between the largest eigenvalues is

$$
\begin{aligned}
\lambda_{1}(A)-\lambda_{1}\left(A_{m}\right)= & x_{1}^{T} A_{m} x_{1}-w_{1}^{T} A_{m} w_{1} \\
& +2 \sum_{l \in \mathcal{M}_{m}}\left(x_{1}\right)_{l^{+}}\left(x_{1}\right)_{l^{-}},
\end{aligned}
$$

which, since $A_{m}$ and $A$ are symmetric, also can be written as

$$
\begin{aligned}
\lambda_{1}(A)-\lambda_{1}\left(A_{m}\right)= & \left(x_{1}-w_{1}\right)^{T} A_{m}\left(x_{1}+w_{1}\right) \\
& +2 \sum_{l \in \mathcal{M}_{m}}\left(x_{1}\right)_{l^{+}}\left(x_{1}\right)_{l^{-}}
\end{aligned}
$$

or as

$$
\begin{aligned}
\lambda_{1}(A)-\lambda_{1}\left(A_{m}\right)= & \left(x_{1}-w_{1}\right)^{T} A\left(x_{1}+w_{1}\right) \\
& +2 \sum_{l \in \mathcal{M}_{m}}\left(w_{1}^{T}\right)_{l^{+}}\left(w_{1}\right)_{l^{-}} .
\end{aligned}
$$

The Perron-Frobenius theorem [3] implies that there is at least one component in $x_{1}-w_{1}$ that is negative (because $x_{1}^{T} x_{1}=$ $\left.w_{1}^{T} w_{1}=1\right)$.

The expansions (10) and (11) should be compared with the exact expressions (12) and (13), respectively. Moreover, they lead to a second proof of Lemma 4, provided a second-order perturbation is accurate enough. Since the sum in (11), as well as in (10), is positive and all components $w_{1}$ and $x_{1}$ are positive when $G$ is connected, comparison with (12) and (13) suggests (provided a second-order perturbation is accurate enough), for connected graphs, that

$$
\left(x_{1}-w_{1}\right)^{T} A\left(x_{1}+w_{1}\right)>0,
$$

while

$$
\left(x_{1}-w_{1}\right)^{T} A_{m}\left(x_{1}+w_{1}\right)<0 .
$$

For large graphs, where $\lambda_{1}=O(N)$, the expansion up to second order, thus ignoring terms of $O\left(\zeta^{3}\right)$ in (8) and (9), can already be good. This is the approach of Restreppo et al. [4] and verified numerically by Milanese et al. [5].

\section{B. Closed walks in subgraphs}

Let $G$ be a connected graph with adjacency matrix $A$. From the decomposition [3], art. 156 on p. 226]

$$
A=\sum_{i=1} \lambda_{i} x_{i} x_{i}^{T}
$$

using $x_{i}^{T} x_{j}=0$ for $i \neq j$ and $x_{i}^{T} x_{i}=1$ for any $i$, we have that

$$
A^{k}=\sum_{i=1}^{n} \lambda_{i}^{k} x_{i} x_{i}^{T}
$$

When $k \rightarrow \infty$, the most important term in the sum above is $\lambda_{1}^{k} x_{1} x_{1}^{T}$, provided that $G$ is nonbipartite. ${ }^{3}$ In such a case, we have $\lambda_{1}>\left|\lambda_{i}\right|$ for $i=2, \ldots, n$, and so, for any two nodes $u, v$ of $G$,

$$
\begin{aligned}
\lim _{k \rightarrow \infty} \frac{\left(A^{k}\right)_{u v}}{\lambda_{1}^{k}\left(x_{1}\right)_{u}\left(x_{1}\right)_{v}} & =\lim _{k \rightarrow \infty} \frac{\sum_{i=1}^{n} \lambda_{i}^{k}\left(x_{i}\right)_{u}\left(x_{i}\right)_{v}}{\lambda_{1}^{k}\left(x_{1}\right)_{u}\left(x_{1}\right)_{v}} \\
& =1+\sum_{i=2}^{n} \frac{\left(x_{i}\right)_{u}\left(x_{i}\right)_{v}}{\left(x_{1}\right)_{u}\left(x_{1}\right)_{v}}\left(\frac{\lambda_{i}}{\lambda_{1}}\right)^{k}=1 .
\end{aligned}
$$

In view of the above, we will deliberately resort to the following approximation:

$$
\text { For large } k:\left(A^{k}\right)_{u v} \approx \lambda_{1}^{k}\left(x_{1}\right)_{u}\left(x_{1}\right)_{v} .
$$

Under such approximation, the total number of closed walks of large length $k$ in $G$ is

$$
\sum_{u \in V(G)}\left(A^{k}\right)_{u u} \simeq \sum_{u \in V(G)} \lambda_{1}^{k}\left(x_{1}\right)_{u}\left(x_{1}\right)_{u}=\lambda_{1}^{k} \sum_{u \in V(G)}\left(x_{1}\right)_{u}^{2}=\lambda_{1}^{k} .
$$

We will demonstrate that removing the node $u$ or the link $u \sim v$ with highest vector component $\left(x_{1}\right)_{u}$ or highest vector component product $\left(x_{1}\right)_{u}\left(x_{1}\right)_{v}$ will decrease $\lambda_{1}(A)$ most.

\section{Node removal}

In order to find the node whose deletion reduces $\lambda_{1}$ most, we will consider the equivalent question: Which deleted node $u$ reduces the number of closed walks in $G$ for some large length $k$ most?

Of course, the number of closed walks of length $k$ that start at node $u$ is equal to $\left(A^{k}\right)_{u u} \approx \lambda_{1}^{k}\left(x_{1}\right)_{u}^{2}$. When we delete node $u$ from $G$, then, besides the closed walks that start at $u$, we also destroy the closed walks that start at another node $v$, but

${ }^{3}$ In case $G$ is bipartite, let $(U, V)$ be the bipartition of nodes of $G$. Then $\lambda_{n}=-\lambda_{1},\left(x_{n}\right)_{u}=\left(x_{1}\right)_{u}$ for $u \in U$ and $\left(x_{n}\right)_{v}=-\left(x_{1}\right)_{v}$ for $v \in V$. Both $\lambda_{1}$ and $\lambda_{n}$ are simple eigenvalues, so that $\lambda_{1}>\left|\lambda_{i}\right|$ for $i=2, \ldots, n-1$. Similarly as above we get

$$
\lim _{k \rightarrow \infty} \frac{\left(A^{k}\right)_{u, v}}{\lambda_{1}^{k}\left(x_{1}\right)_{u}\left(x_{1}\right)_{v}}=1+\lim _{k \rightarrow \infty}(-1)^{k} \frac{\left(x_{n}\right)_{u}\left(x_{n}\right)_{v}}{\left(x_{1}\right)_{u}\left(x_{1}\right)_{v}} .
$$

Obviously, the limit above exists if we restrict $k$ to range over odd or even numbers only, in which case the limit is either 0 or 2, depending on whether $u$ and $v$ belong to the same or different parts of the bipartition. This suggests that the same strategy will extend to bipartite graphs as well, except that the argument will have to take into account the nonexistence of odd closed walks. 
that contain $u$ as well. Any such closed walk that starts at $v$ may contain several occurrences of $u$.

For fixed $u, k$, and $v$, let $W_{t}$ denote the number of closed walks of length $k$ that start at $v$ and that contain $u$ at least $t$ times, $t \geqslant 1$. Suppose that in such a walk, node $u$ appears after $l_{1}$ steps, after $l_{1}+l_{2}$ steps, after $l_{1}+l_{2}+l_{3}$ steps, and so on, the last appearance counted after $l_{1}+\cdots+l_{t}$ steps. Here $l_{1}, \ldots, l_{t} \geqslant 1$. Moreover, $u$ must appear for the last time after at most $k-1$ steps (after $k$ steps we are back at $v$ ); thus we may also introduce $l_{t+1}=k-\left(l_{1}+\cdots+l_{t}\right)$ and ask that $l_{t+1} \geqslant 1$. Then we have

$$
\begin{aligned}
W_{t} & =\sum_{l_{1}, \ldots, l_{t+1}}\left(A^{l_{1}}\right)_{v u}\left(A^{l_{2}}\right)_{u u} \cdots\left(A^{l_{t}}\right)_{u u}\left(A^{l_{t+1}}\right)_{u v} \\
& \simeq \sum_{l_{1}, \ldots, l_{t+1}} \lambda_{1}^{k}\left(x_{1}\right)_{v}^{2}\left(x_{1}\right)_{u}^{2 t}=\lambda_{1}^{k}\left(x_{1}\right)_{v}^{2}\left(x_{1}\right)_{u}^{2 t} \sum_{\sum_{j=1}^{t+1} l_{j}=k ; l_{j} \geqslant 1} 1 .
\end{aligned}
$$

Introducing $l_{1}^{\prime}=l_{1}-1, \ldots, l_{t+1}^{\prime}=l_{t+1}-1$, the last sum is equal to the number of nonnegative solutions to

$$
l_{1}^{\prime}+l_{2}^{\prime}+\cdots+l_{t}^{\prime}+l_{t+1}^{\prime}=k-t-1,
$$

which is, in turn, equal to $\left(\begin{array}{c}(k-1-t)+t \\ t\end{array}\right)=\left(\begin{array}{c}k-1 \\ t\end{array}\right)$. Therefore,

$$
W_{t} \simeq\left(\begin{array}{c}
k-1 \\
t
\end{array}\right) \lambda_{1}^{k}\left(x_{1}\right)_{v}^{2}\left(x_{1}\right)_{u}^{2 t} .
$$

Consider now a closed walk of length $k$ starting at $v$, which contains $u$ exactly $j$ times. Such a walk is counted $j$ times in $W_{1},\left(\begin{array}{l}j \\ 2\end{array}\right)$ times in $W_{2},\left(\begin{array}{l}j \\ 3\end{array}\right)$ times in $W_{3}, \ldots,\left(\begin{array}{l}j \\ j\end{array}\right)$ times in $W_{j}$, and using the well-known equality

$$
1=\sum_{t \geqslant 1}(-1)^{t-1}\left(\begin{array}{l}
j \\
t
\end{array}\right),
$$

we see that this closed walk is counted exactly once in the expression

$$
W^{v}=W_{1}-W_{2}+W_{3}-\cdots+(-1)^{t-1} W_{t}+\cdots .
$$

Thus, $W^{v}$ represents the number of closed walks of length $k$ starting at $v$, which will be affected by deleting $u$. From the above expression for $W_{t}$, we have

$$
\begin{aligned}
W^{v} & \simeq \sum_{t \geqslant 1}(-1)^{t-1}\left(\begin{array}{c}
k-1 \\
t
\end{array}\right) \lambda_{1}^{k}\left(x_{1}\right)_{v}^{2}\left(x_{1}\right)_{u}^{2 t} \\
& =-\lambda_{1}^{k}\left(x_{1}\right)_{v}^{2} \sum_{t \geqslant 1}\left(\begin{array}{c}
k-1 \\
t
\end{array}\right)\left[-\left(x_{1}\right)_{u}^{2}\right]^{t} \\
& =\lambda_{1}^{k}\left(x_{1}\right)_{v}^{2}\left\{1-\left[1-\left(x_{1}\right)_{u}^{2}\right]^{k-1}\right\} .
\end{aligned}
$$

Therefore, the total number of closed walks of length $k$ destroyed by deleting $u$ is equal to

$$
\begin{aligned}
W & \simeq \lambda_{1}^{k}\left(x_{1}\right)_{u}^{2}+\sum_{v \neq u} W^{v} \\
& =\lambda_{1}^{k}\left(x_{1}\right)_{u}^{2}+\lambda_{1}^{k} \sum_{v \neq u}\left(x_{1}\right)_{v}^{2}\left\{1-\left[1-\left(x_{1}\right)_{u}^{2}\right]^{k-1}\right\} \\
& =\lambda_{1}^{k}\left(\left(x_{1}\right)_{u}^{2}+\left[1-\left(x_{1}\right)_{u}^{2}\right]\left\{1-\left[1-\left(x_{1}\right)_{u}^{2}\right]^{k-1}\right\}\right) \\
& =\lambda_{1}^{k}\left(1-\left[1-\left(x_{1}\right)_{u}^{2}\right]^{k}\right) .
\end{aligned}
$$

The last function is increasing in $\left(x_{1}\right)_{u}$ in the interval $[0,1]$, and so we conclude that most closed walks are destroyed when we remove the node with the largest principal eigenvector component. Hence, the spectral radius [see (5)] is decreased the most in such a case as well.

\section{Link removal}

Similarly as in the previous section, we want to find out the deletion of which link $u \sim v$ mostly reduces the number of closed walks in $G$ of some large length $k$ ?

For fixed $u, v$, and $k$, let $W_{t}$ denote the number of closed walks of length $k$ that start at some node $w$ and contain the link $u \sim v$ at least $t$ times, $t \geqslant 1$. Suppose that in such a walk, the link $u \sim v$ appears at positions $1 \leqslant l_{1} \leqslant l_{2} \leqslant \cdots \leqslant l_{t} \leqslant k$ in the sequence of links on the walk, and let $u_{i, 0}$ and $u_{i, 1}$ be the first and the second nodes of the $i$ th appearance of $u v$ in the walk. Obviously, either $\left(u_{i, 0}, u_{i, 1}\right)=(u, v)$ or $\left(u_{i, 0}, u_{i, 1}\right)=$ $(v, u)$. Then

$$
\begin{aligned}
W_{t}= & \sum_{w \in V} \sum_{l_{1} \leqslant \cdots \leqslant l_{t}}\left(A^{l_{1}-1}\right)_{w u_{1,0}} \\
& \times\left[\prod_{i=2}^{t}\left(A^{l_{i}-l_{i-1}-1}\right)_{u_{i-1,1} u_{i, 0}}\right]\left(A^{k-l_{t}-1}\right)_{u_{t, 1} w} \\
\simeq & \sum_{w \in V} \sum_{l_{1} \leqslant \cdots \leqslant l_{t}} \lambda_{1}^{l_{1}-1}\left(x_{1}\right)_{w}\left(x_{1}\right)_{u_{1,0}} \\
& \times\left[\prod_{i=2}^{t} \lambda_{1}^{l_{i}-l_{i-1}-1}\left(x_{1}\right)_{u_{i-1,1}}\left(x_{1}\right)_{u_{i, 0}}\right] \lambda_{1}^{k-l_{t}-1}\left(x_{1}\right)_{u_{t, 1}}\left(x_{1}\right)_{w} \\
= & \sum_{w \in V}\left(x_{1}\right)_{w}^{2} \sum_{l_{1} \leqslant \cdots \leqslant l_{t}} \lambda_{1}^{k-t} \prod_{i=1}^{t}\left[\left(x_{1}\right)_{u_{i, 0}}\left(x_{1}\right)_{u_{i, 1}}\right]^{2} \\
= & \left(\begin{array}{l}
k \\
t
\end{array}\right) \lambda_{1}^{k-t}\left(2\left(x_{1}\right)_{u}\left(x_{1}\right)_{v}\right)^{t} .
\end{aligned}
$$

The term $2\left(x_{1}\right)_{u}\left(x_{1}\right)_{v}$ appears in the last equation because there are two ways to choose $\left(x_{u_{i, 0}}, x_{u_{i, 1}}\right)$ for each $i=1, \ldots, t$.

Now, the number of walks affected by deleting the link $u \sim v$ is equal to

$$
\begin{aligned}
W^{u v} & =\sum_{t \geqslant 1}(-1)^{t-1} W_{t} \\
& =\sum_{t \geqslant 1}(-1)^{t-1}\left(\begin{array}{l}
k \\
t
\end{array}\right) \lambda_{1}^{k-t}\left[2\left(x_{1}\right)_{u}\left(x_{1}\right)_{v}\right]^{t} \\
& =\lambda_{1}^{k}-\sum_{t \geqslant 0}(-1)^{t}\left(\begin{array}{l}
k \\
t
\end{array}\right) \lambda_{1}^{k-t}\left[2\left(x_{1}\right)_{u}\left(x_{1}\right)_{v}\right]^{t} \\
& =\lambda_{1}^{k}-\left[\lambda_{1}-2\left(x_{1}\right)_{u}\left(x_{1}\right)_{v}\right]^{k} .
\end{aligned}
$$

The last function is increasing in $\left(x_{1}\right)_{u}\left(x_{1}\right)_{v}$ in the interval $\left[0, \lambda_{1} / 2\right]$, and so most closed walks are destroyed when we remove the link with the largest product of principal eigenvector components. Thus, the spectral radius is decreased the most in such a case as well.

\section{Assortativity and lower bounds for $\lambda_{1}$}

A lower bound of the largest adjacency eigenvalue $\lambda_{1} \geqslant \frac{N_{3}}{N_{2}}$ has been proved in Ref. [18], where $N_{k}$ is the total number of 
walks of length $k$. The lower bound $\frac{N_{3}}{N_{2}}$ appeared earlier as an approximation in Ref. [19] of the largest adjacency eigenvalue $\lambda_{1}$, and it is a perfect linear function of assortativity $\rho_{D}$ [18].

Let us first look at the decrease of $\frac{N_{3}}{N_{2}}$ by a link removal. We know [18] that

$$
\frac{N_{3}}{N_{2}}=\frac{\sum_{i=1}^{N} d_{i}^{3}-\sum_{i \sim j}\left(d_{i}-d_{j}\right)^{2}}{\sum_{i=1}^{N} d_{i}^{2}} .
$$

We denote $N_{3}$ and $N_{3}^{\prime}$ as the number of three hop walks in the original graph $G$ and in the graph $G \backslash\left\{l_{i j}\right\}$ with one link $l=i \sim j$ less, respectively. Then we have that

$$
\begin{aligned}
\Delta_{3}= & N_{3}-N_{3}^{\prime} \\
= & d_{i}^{3}+d_{j}^{3}-\left(d_{i}-1\right)^{3}-\left(d_{j}-1\right)^{3}-\left(d_{i}-d_{j}\right)^{2} \\
& -\sum_{l \in \mathcal{N}(i), l \neq j}\left(d_{l}-d_{i}\right)^{2}-\left(d_{l}-d_{i}+1\right)^{2} \\
& -\sum_{l \in \mathcal{N}(j), l \neq i}\left(d_{l}-d_{j}\right)^{2}-\left(d_{l}-d_{j}+1\right)^{2},
\end{aligned}
$$

where $d_{i}$ is the degree of node $i$ in the original graph $G$ and $\mathcal{N}(i)$ is the set of the neighbors of node $i$. The decrease $\Delta_{3}$ can be simplified as

$$
\begin{aligned}
\Delta_{3}= & 2-3\left(d_{i}+d_{j}\right)+3\left(d_{i}^{2}+d_{j}^{2}\right)-\left(d_{i}-d_{j}\right)^{2} \\
& +\sum_{l \in \mathcal{N}(i), k \neq j}\left(2 d_{l}-2 d_{i}+1\right)+\sum_{l \in \mathcal{N}(j), l \neq i}\left(2 d_{l}-2 d_{j}+1\right) \\
= & 2\left(d_{i}^{2}+d_{j}^{2}\right)+2 d_{i} d_{j}+2-3\left(d_{i}+d_{j}\right) \\
& +\left(d_{i}+d_{j}-2\right)-2 d_{i}\left(d_{i}-1\right)-2 d_{j}\left(d_{j}-1\right) \\
& +\sum_{l \in \mathcal{N}(i), k \neq j} 2 d_{l}+\sum_{l \in \mathcal{N}(j), k \neq i} 2 d_{l} \\
= & 2 d_{i} d_{j}+\sum_{l \in \mathcal{N}(i), k \neq j} 2 d_{l}+\sum_{l \in \mathcal{N}(j), k \neq i} 2 d_{l} \\
= & 2 d_{i} d_{j}+2\left(s_{i}+s_{j}\right)-2\left(d_{i}+d_{j}\right),
\end{aligned}
$$

where

$$
s_{i}=\sum_{l \in \mathcal{N}(i)} d_{l}
$$

is the total degree of all the direct neighbors of a node $i$. Similarly, the decrease in the number of two hop walks is denoted as

$$
\Delta_{2}=N_{2}-N_{2}^{\prime}=2\left(d_{i}+d_{j}-1\right) .
$$

Note that $\Delta_{2}$ and $\Delta_{3}$ are only functions of a local property, i.e., the degree $d_{i}$ and $d_{j}$ of the two end nodes of a link $l_{i j}$. The complexity of computing $\Delta_{3}$ or $\Delta_{2}$ for all linked node pairs is $O\left(N^{2}\right)$ in a dense graph, which is the worst case.

\section{STRATEGIES TO MINIMIZE THE LARGEST EIGENVALUE BY LINK REMOVAL}

This section discusses and compares various strategies in Fig. 5, denoted by $S$.

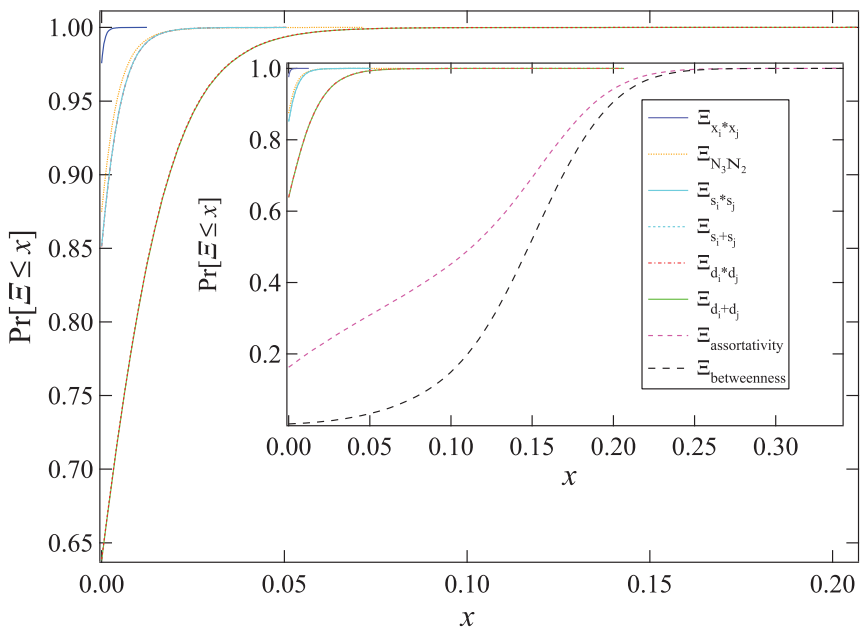

FIG. 5. (Color online) Various strategies applied to $10^{6}$ instances of ER graphs with $N=20$ and $p=2 \ln N / N$. The insert shows two additional strategies "assortativity" and "betweenness" that are clearly worse than the others.

The first strategy, as suggested in Sec. III, is to remove the link with maximum product of the eigenvector components. Specifically, this strategy is denoted by $S=x_{i} x_{j}$ instead of $S=\left(x_{1}\right)_{i}\left(x_{1}\right)_{j}$ to simplify the notation in the figures, and it removes that link $l=i \sim j$ for which $\left(x_{1}\right)_{i}\left(x_{1}\right)_{j}$ is maximal.

Section IIIC hints that the spectral radius is possibly decreased the most by a link removal that reduces either $S=\frac{N_{3}}{N_{2}}$ or the assortativity $S=\rho_{D}$ the most. Strategy $S=\frac{N_{3}}{N_{2}}$ will remove the link such that $\frac{N_{3}-\Delta_{3}}{N_{2}-\Delta_{2}}$ is minimized.

The other considered strategies $S=d_{i} d_{j}$ and $S=d_{i}+d_{j}$ remove that link $l=i \sim j$ with the largest sum or product of the degrees of the link's end points, whereas the strategies $S=s_{i}+s_{j}$ and $S=s_{i} s_{j}$ remove the link with the largest sum or product of the total degree $s_{i}$ of the neighbors at both end points. Finally, we also considered the strategy $S=$ betweenness, which removes the link with highest link betweenness, i.e., the number of shortest paths between all node pairs that traverse the link.

We define the performance measure $\Xi_{S}$ of a particular link removal strategy $S$ by

$$
\Xi_{S}=\left[\lambda_{1}(A)-\lambda_{1}\left(A_{1}\right)\right]_{\text {optimal }}-\left[\lambda_{1}(A)-\lambda_{1}\left(A_{1}\right)\right]_{\text {Strategy } S} .
$$

Figure 5 compares the above explained strategies. Figure 5 confirms that strategy $S=x_{i} x_{j}$ is superior to all other strategies. There is a very small difference between the strategies $S=d_{i}+d_{j}$ and $S=d_{i} d_{j}$ and between $S=s_{i}+s_{j}$ and the corresponding product $S=s_{i} s_{j}$. In both cases the product strategy is slightly better (but the difference is not observable from Fig. 5).

Another strategy is to remove the link that possibly disconnects the graph $G$ into two disjoint graphs $G_{1}$ and $G_{2}$. However, this strategy is not always optimal as illustrated in Fig. 6.

Only when both $G_{1}$ and $G_{2}$ are the same did we find that the removal of the connecting link induces the largest decrease in $\Delta \lambda_{1}$. Since this strategy cannot always be applied, we have ignored this strategy henceforth. 


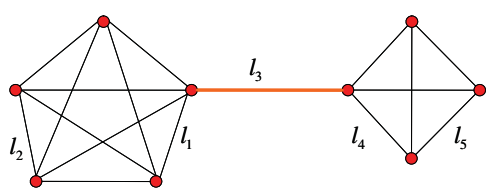

$\Delta \lambda_{1}=\lambda_{1}(A)-\lambda_{1}\left(A \backslash l_{1}\right)=0.3486$

$\Delta \lambda_{1}=\lambda_{1}(A)-\lambda_{1}\left(A \backslash l_{2}\right)=0.3145$

$\Delta \lambda_{1}=\lambda_{1}(A)-\lambda_{1}\left(A \backslash l_{3}\right)=0.0806$

$\Delta \lambda_{1}=\lambda_{1}(A)-\lambda_{1}\left(A \backslash l_{4}\right)=0.0167$

$\Delta \lambda_{1}=\lambda_{1}(A)-\lambda_{1}\left(A \backslash l_{5}\right)=0.0092$

FIG. 6. (Color online) All possible $\Delta \lambda_{1}$ are computed when one link is removed.

\section{A. Removing $m>1$ links}

In this section, we investigate the behavior of several strategies when more than one link is removed. We generated $10^{4}$ Erdős-Rényi graphs with $N=10$ nodes and $L=20$ links, of which about $2 \%$ are disconnected. From each of the generated graphs, all the links are removed one by one following the different "greedy" strategies. We compare the decrease in $\lambda_{1}$ for each strategy to the optimal solution found by removing all possible combinations of $m$ links. In Fig. 7 the percentage of agreement between the greedy strategies and the optimal strategy is shown.

Figure 7 illustrates that strategy $S=\max _{1 \leqslant(i, j) \leqslant N}\left(x_{1}\right)_{i}\left(x_{1}\right)_{j}$ is nearly always (except for $m=13$ ) superior to strategy $S=$ $N_{3} / N_{2}$ and $S=s_{i} s_{j}$, which agrees with the theory in Sec. III. Figure 7 exhibits a regime change from $m=10$ on, where the connectivity of the graphs starts to decrease rapidly.

The peculiar regime for $m>10$ can be understood as follows. The optimal solution for $m=10$ removals is a circuit, if the original graph contains a single connected circuit on $N=10$ nodes. If strategy $S=\max _{1 \leqslant(i, j) \leqslant N}\left(x_{1}\right)_{i}\left(x_{1}\right)_{j}$ finds the optimal solution for $m=10$ removals, the only possible solution for $m=11$ removals is to cut the circuit to form a path. This is also the optimal solution. The eigenvector components of a path graph are symmetrical around the node(s) in the middle of the path and are maximal for the center node(s). Strategy $S=\max _{1 \leqslant(i, j) \leqslant N}\left(x_{1}\right)_{i}\left(x_{1}\right)_{j}$ will, for the next link removal, cut the path in the middle. The resulting graph is also the optimal solution. In the next step, however, the strategy will cut one of the paths in two, resulting in three paths of lengths one, two, and four links, respectively. The optimal solution for $m=13$ link removals consists of a graph with three paths of

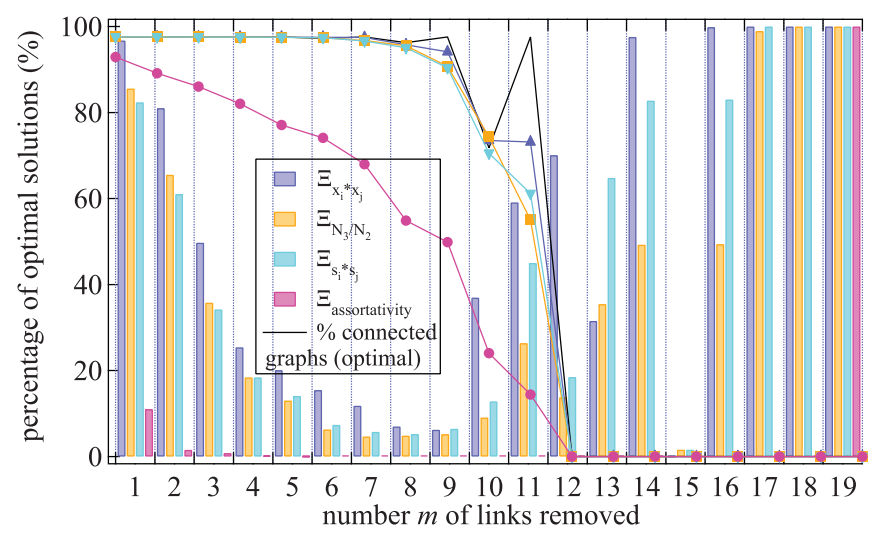

FIG. 7. (Color online) Four strategies compared with the global optimum as a function of the number $m$ of removed links in ER random graphs with $N=10$ nodes and $L=20$ links, where $10^{4}$ instances are generated. The lines show the percentage of connected graphs per strategy after the removal of $m$ links. lengths two and one of length three. This graph can never be formed by strategy $S=\max _{1 \leqslant(i, j) \leqslant N}\left(x_{1}\right)_{i}\left(x_{1}\right)_{j}$ starting from a circuit. The optimal solution for $m=14$ consists of two paths of length two and two paths of length one, which can be obtained in many different ways, including cutting the longest path of the solution for $m=13$. In almost $98 \%$ of the cases this solution is found by strategy $S=\max _{1 \leqslant(i, j) \leqslant N}\left(x_{1}\right)_{i}\left(x_{1}\right)_{j}$. The high success rate means, at the same time, that the optimal solution for $m=15$ is almost never found because it cannot be reached from the optimal solution of $m=14$ by another link removal, regardless of the followed strategy. The weaker performance of strategy $S=s_{i} s_{j}$ for $m=12$ can be explained by considering the optimal solution for $m=11$, which is a path of nine links. Strategy $S=s_{i} s_{j}$ removes the link that has the maximum product of the one hop neighbors of its endpoints. Since a path has an even degree distribution, except for the endpoints, the five links that form the center of the path have an equal probability of being removed. Consequently, the optimal solution for $m=11$ will result in the optimal solution for $m=12$ only one in five times. The other four possibilities lead to a graph with either a combination of a path of length two and a path of length six or a combination of a path of length three and a path of length five. Both these graphs will give the optimal solution for $m=13$ link removals, which explains the increased success rate for $m=13$.

At $m=15$, the graph consists of five links and $N=$ 10 nodes, configured in separate "cliques" $K_{2}$ (i.e., line segments), and the largest eigenvalue is minimal at $\lambda_{1}=1$. For $m>15$, the strategies are all the same: A clique $K_{2}$ (i.e., disjoint link) is removed.

Figure 8 illustrates four strategies on a typical instance of a network with $N=10$ and $L=20$ links. While the strategy $S=$ assortativity clearly underperforms, the three other strategies $S=x_{i} x_{j}, S=N_{3} / N_{2}$, and $S=s_{i} s_{j}$ are competitive: For small $m$, the strategy $S=x_{i} x_{j}$ excels (as shown in Fig. 7), but for larger $m$ the others can outperform. Again, this phenomenon is characteristic for an NP-complete problem, where the whole previous history of links removals affects the current link removal. The considered strategies (except for the global optimum one) are greedy and optimize only the current link removal, irrespective of the way in which the current graph $G_{m}$ is obtained previously.

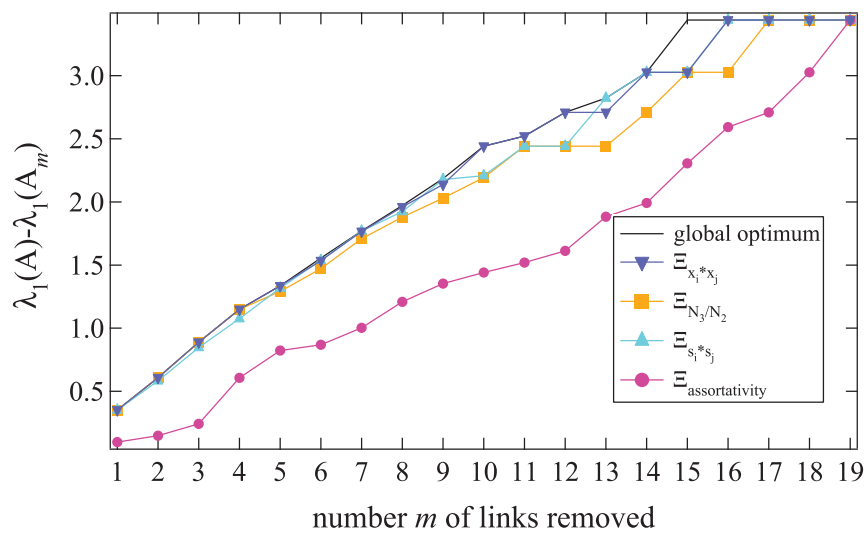

FIG. 8. (Color online) The performance $\lambda_{1}(A)-\lambda_{1}\left(A_{m}\right)$ of four strategies versus $m$ link removals in a typical instance of a graph with $N=10$ and $L=20$ links. 


\section{SCALING LAW OF $\left(\lambda_{1}(A)-\lambda_{1}\left(A_{m}\right)\right)_{\text {optimal }}$}

Another observation from Fig. 8 is that

$$
\left.\Delta \lambda_{m}\right|_{\text {optimal }}=\lambda_{1}(A)-\left.\lambda_{1}\left(A_{m}\right)\right|_{\text {optimal }}=O\left(m^{\beta}\right),
$$

where $\beta \leqslant 1$. In other words, we conjecture that the scaling of $\lambda_{1}(A)-\lambda_{1}\left(A_{m}\right)$ with $m$ is sublinear in $m$ (for nonregular graphs) and that the coefficient $\beta$ is likely a function of the type of graph. Obviously, $\Delta \lambda_{m}=0$, when $m=0$. Applying the upper bound (1) to $\lambda_{1}\left(A_{m}\right)$ shows that

$$
\begin{aligned}
\Delta \lambda_{m} & \geqslant \lambda_{1}(A)-\sqrt{1-\frac{1}{N}} \sqrt{2 L-2 m} \\
& \geqslant \lambda_{1}(A)-\sqrt{1-\frac{1}{N}} \sqrt{2 L}+\sqrt{1-\frac{1}{N}} \sqrt{2 m}=O\left(m^{1 / 2}\right) .
\end{aligned}
$$

On the other hand, if $G_{m}$ is a regular graph, then

$$
\Delta \lambda_{m}=\lambda_{1}(A)-\frac{2 L-2 m}{N}=O(m) .
$$

In particular, if $G$ and $G_{m}$ are regular graphs, then

$$
\Delta \lambda_{m}=\frac{2 m}{N} \text {. }
$$

These arguments illustrate that $\frac{1}{2}<\beta \leqslant 1$. Figure 8 shows that $\lambda_{1}(A)-\left.\lambda_{1}\left(A_{m}\right)\right|_{\text {optimal }}$ is likely close to $\beta=1$, which suggests that the optimal way to remove $m$ links is to make $G_{m}$ as regular as possible, because the lowest possible $\lambda_{1}\left(A_{m}\right)$ with given $N$ and $L-m$ is obtained for a regular graph [as follows from the Rayleigh inequality $\left.\lambda_{1}(A) \geqslant \frac{2 L}{N}\right]$.

While the law (15) is difficult to prove in general, we provide evidence by computing the decrease in $\lambda_{1}$ when $m$ random links are removed in the class of Erdôs-Rényi random graphs $G_{p}(N)$. For sufficiently large Erdős-Rényi random graphs $G_{p}(N)$, we know [3] that

$$
E\left[\lambda_{1}\right]=(N-2) p+1+O\left(\frac{1}{\sqrt{N}}\right) .
$$

When $m$ random links are removed from $G_{p}(N)$, we again obtain an Erdôs-Rényi random graph with link density

$$
p^{*}=\frac{L-m}{\left(\begin{array}{c}
N \\
2
\end{array}\right)} \text {. }
$$

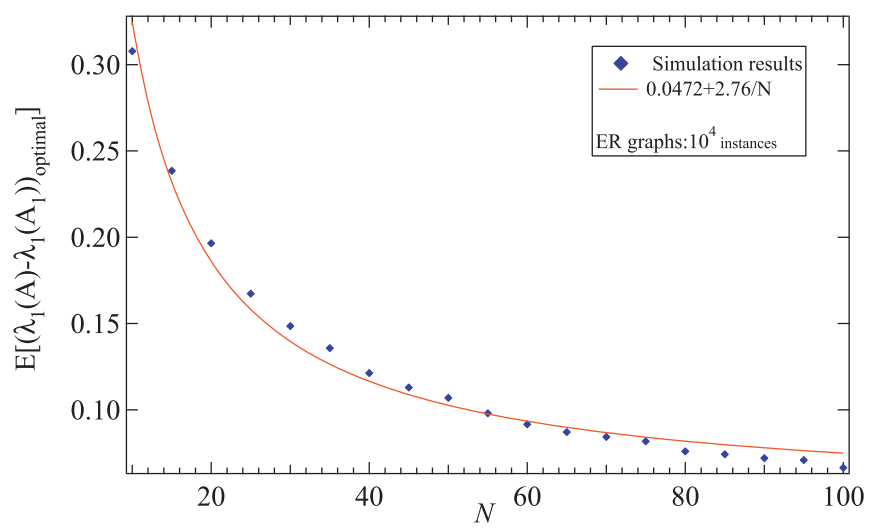

FIG. 9. (Color online) The scaling law of $\left(\lambda_{1}(A)-\lambda_{1}\left(A_{1}\right)\right)_{\text {optimal }}$ for ER random graphs as a function of $N$.

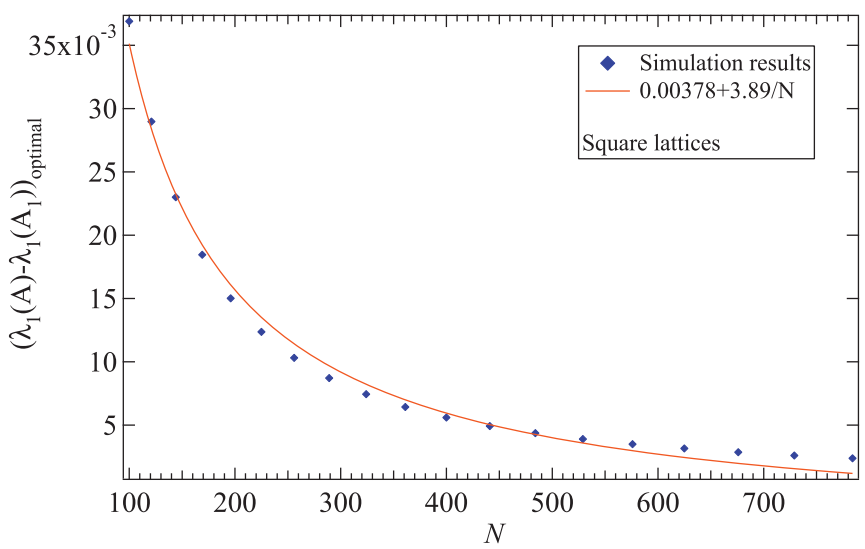

FIG. 10. (Color online) The scaling law of $\left(\lambda_{1}(A)-\lambda_{1}\left(A_{1}\right)\right)_{\text {optimal }}$ for square lattices as a function of $N$.

Hence,

$$
\begin{aligned}
E\left[\Delta \lambda_{m}\right] & =E\left[\lambda_{1}\left(G_{p}(N)\right)\right]-E\left[\lambda_{1}\left(G_{p^{*}}(N)\right)\right] \\
& =(N-2)\left(p-p^{*}\right)+R_{p}(N),
\end{aligned}
$$

where the error term $R_{p}(N)$ is unknown. Assuming that $R_{p}(N)$ is negligibly small, we find, for sufficiently high $N$,

$$
E\left[\Delta \lambda_{m}\right] \simeq \frac{(N-2) m}{\left(\begin{array}{c}
N \\
2
\end{array}\right)}=\frac{2 m}{N}-\frac{2 m}{N(N-1)} .
$$

Thus, the average decrease in $\lambda_{1}(A)-\lambda_{1}\left(A_{m}\right)$ after removing $m$ random links in $G_{p}(N)$ is approximately, for large $N$,

$$
E\left[\Delta \lambda_{m}\right] \simeq \frac{2 m}{N},
$$

which is close to (16) for regular graphs.

For $m=1$, simulations on various types of graphs in Figs. 9 and 10 suggest the scaling law

$$
\left(\lambda_{1}(A)-\lambda_{1}\left(A_{1}\right)\right)_{\mathrm{optimal}}=\frac{\alpha}{N},
$$

where $\alpha$ is graph specific. In other words, $N \Delta \lambda_{1}=\alpha$ is independent of the size of the graph.

Ignoring the asymptotic nature of the analysis that led to (17), we observe that, for $m=1$, a maximum occurs at $N=2$. Figure 11 shows the pdf of $\Delta \lambda$ for Erdős-Rényi random graphs, where for each curve $10^{6} \mathrm{ER}$ graphs have been created

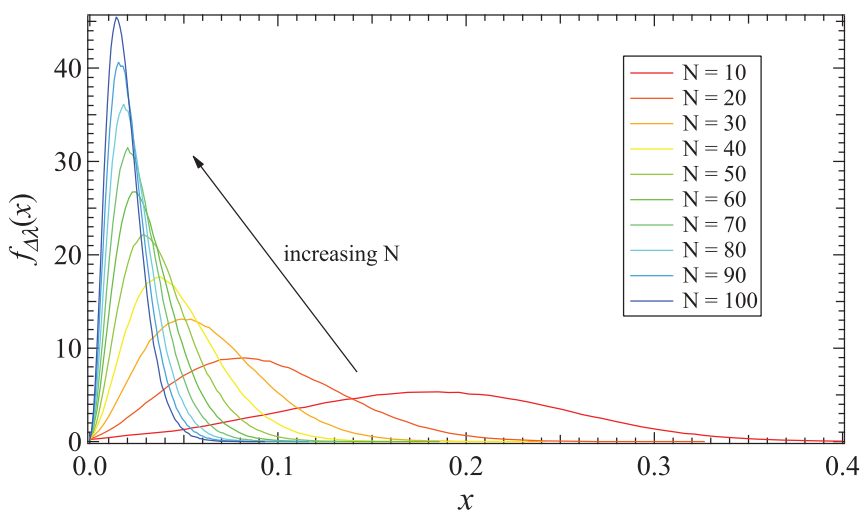

FIG. 11. (Color online) The probability density function of $\Delta \lambda_{1}$ for ER random graphs of several sizes $N$. 
in which one random link was removed. The simulations agree with $E[\Delta \lambda] \simeq \frac{2}{N}$ and indicate that $\operatorname{Var}[\Delta \lambda] \simeq 13+2 E[\Delta \lambda]$. Since a random link removal is inferior to the removal of the optimal link, Fig. 9 indeed illustrates that the coefficient of the inverse $N$ scaling law $\alpha_{G_{p}(N)} \simeq 2.75>2$. Figure 10 shows that $\alpha_{\text {lattice }} \simeq 3.9>\alpha_{G_{p}(N)} \simeq 2.75$, which may indicate that deviations from regularity cause $\lambda_{1}$ to decrease more.

\section{CONCLUSIONS}

The spectral radius is both fundamental in graph theory as well as in many dynamic processes in complex networks such as epidemic spreading, synchronization, and reaching consensus [3], p. 200]. We have shown that the spectral radius minimization problem (for both link as node removals) is an NP-hard problem, which opens the race to find the best heuristic. In particular, in large infrastructures such as transportation networks, where removing links can be very costly, a near to optimal strategy is desirable. We have shown that an excellent strategy is $S=x_{i} x_{j}$ : On average, this strategy outperforms most other heuristics, but it does not beat them at all times. In addition to graph theoretic bounds and arguments that underline the goodness of the heuristic $S=x_{i} x_{j}$, two scaling laws (15) and (18) are found: These laws may help to estimate the decrease in spectral radius as a function of the number $N$ of nodes and/or the number $m$ of link removals. It may be worthwhile that further investigations compute or estimate the scaling parameters $\beta$ in (15) as well as $\alpha$ in (18).

\section{ACKNOWLEDGMENTS}

This research was supported by Next Generation Infrastructures (Bsik) and the EU FP7 project ResumeNet (project No. 224619). DS gratefully acknowledges support from Research Project 174033 of the Serbian Ministry of Science and Technological Development and Research Programme P1-0285 of the Slovenian Research Agency.
[1] P. Van Mieghem, J. Omic, and R. E. Kooij, IEEE/ACM Trans. Netw. 17, 1 (2009).

[2] J. G. Restrepo, E. Ott, and B. R. Hunt, Phys. Rev. E 71, 036151 (2005).

[3] P. Van Mieghem, Graph Spectra for Complex Networks (Cambridge University Press, Cambridge, UK, 2011).

[4] J. G. Restrepo, E. Ott, and B. R. Hunt, Phys. Rev. Lett. 97, 094102 (2006).

[5] A. Milanese, J. Sun, and T. Nishikawa, Phys. Rev. E 81, 046112 (2010).

[6] M. R. Garey and D. S. Johnson, Computer and Intractability: A Guide to the Theory of NP-Completeness (W. H. Freeman, New York, 1979).

[7] J. Omic, J. Martin Hernandez, and P. Van Mieghem "Network protection against worms and cascading failures using modularity partitioning", 22nd International Teletraffic Congress (ITC 22), September 7-9, Amsterdam, Netherlands, 2010, available at [http://www.nas.ewi.tudelft.nl/people/Piet/telconference.html].

[8] D. Taylor and J. G. Restrepo, e-print arXiv:1102.4876v1.

[9] T. Watanabe and N. Masuda, Phys. Rev. E 82, 046102 (2010).
[10] T. Nishikawaa and A. E. Motter, Proc. Natl. Acad. Sci. U.S.A. 107, 10342 (2010)

[11] D. M. Cvetković, M. Doob, and H. Sachs, Spectra of Graphs: Theory and Applications, 3rd. ed. (Johann Ambrosius Barth Verlag, Heidelberg, 1995).

[12] N. Robertson and P. D. Seymour, J. Comb. Theory, Ser. B 63, 65 (1995).

[13] Y. Hong, Linear Algebra Appl. 108, 135 (1988).

[14] N. Roussopoulos, Inf. Process. Lett. 2, 108 (1973).

[15] M. Aouchiche, F. K. Bell, D. Cvetković, P. Hansen, P. Rowlinson, S. Simić, and D. Stevanović, Eur. J. Oper. Res. 191, 661 (2008).

[16] D. Stevanović, J. Comb. Theory, Ser. B 91, 143 (2004).

[17] J. H. Wilkinson, The Algebraic Eigenvalue Problem (Oxford University Press, New York, 1965).

[18] P. Van Mieghem, H. Wang, X. Ge, S. Tang, and F. A. Kuipers, Eur. Phys. J. B 76, 643 (2010).

[19] J. G. Restrepo, E. Ott, and B. R. Hunt, Phys. Rev. E 76, 056119 (2007). 